\title{
Representational momentum in dynamic facial expressions is modulated by the level of expressed pain: Amplitude and direction effects
}

\author{
Elise Prigent $^{1}$ • Michel-Ange Amorim ${ }^{2,3}$ • Armando Mónica de Oliveira ${ }^{4}$
}

Published online: 27 September 2017

(C) The Psychonomic Society, Inc. 2017

\begin{abstract}
Humans have developed a specific capacity to rapidly perceive and anticipate other people's facial expressions so as to get an immediate impression of their emotional state of mind. We carried out two experiments to examine the perceptual and memory dynamics of facial expressions of pain. In the first experiment, we investigated how people estimate other people's levels of pain based on the perception of various dynamic facial expressions; these differ both in terms of the amount and intensity of activated action units. A second experiment used a representational momentum (RM) paradigm to study the emotional anticipation (memory bias) elicited by the same facial expressions of pain studied in Experiment 1. Our results highlighted the relationship between the level of perceived pain (in Experiment 1) and the direction and magnitude of memory bias (in Experiment 2): When perceived pain increases, the memory bias tends to be reduced (if positive) and ultimately becomes negative. Dynamic facial expressions of pain may reenact an "immediate perceptual history"
\end{abstract}

Elise Prigent

elise.prigent@limsi.fr

Michel-Ange Amorim

michel-ange.amorim@u-psud.fr

Armando Mónica de Oliveira

1.dinis@ fpce.uc.pt

1 LIMSI, CNRS, Univ. Paris-Sud, Université Paris-Saclay, F-, 91405 Orsay, France

2 CIAMS, Univ. Paris Sud, Université Paris-Saclay, 91405 Orsay Cedex, France

3 CIAMS, Université d'Orléans, 45067 Orléans, France

4 Institute of Cognitive Psychology, University of Coimbra, Coimbra, Portugal in the perceiver before leading to an emotional anticipation of the agent's upcoming state. Thus, a subtle facial expression of pain (i.e., a low contraction around the eyes) that leads to a significant positive anticipation can be considered an adaptive process - one through which we can swiftly and involuntarily detect other people's pain.

Keywords Face perception · Perceptual implicit memory

Facial expressions carry the main visual information used to communicate emotions and intentions to others. As a result, humans have developed a specific capacity to perceive other people's facial expressions so as to get an immediate impression of their emotional state of mind (Palumbo \& Jellema, 2013). These processes are highly adaptive to a world in which we are constantly required to react to, and often anticipate, other people's behavior (Freyd, 1987; Thornton \& Kourtzi, 2002). It has been proposed that, when individuals observe dynamic facial expressions, they involuntarily anticipate how other people's emotional states of mind will unfold in the future, based on the "immediate perceptual history" elicited by these expressions (Jellema, Pecchinenda, Palumbo, \& Tan, 2011). This phenomenon has been referred to an "emotional anticipation" (Jellema et al., 2011) and is thought to reflect the top-down emotional processes that simultaneously allow for a low-level form of mind reading (Palumbo \& Jellema, 2013). Researchers have attempted to more precisely highlight this emotional anticipation through the measurement of a form of perceptual bias known as representational momentum (RM Hubbard, 2014; Thornton, 2014). RM was originally established in studies that involved moving objects, corresponding to the phenomenon that an observer's memory for the final position of a suddenly vanished target is displaced further along the observed 
trajectory it moves (Freyd, 1987; Freyd \& Finke, 1984). This memory bias has also been found to be true for moving human bodies: When a human body is in motion along a trajectory and suddenly vanishes, individuals locate its last perceived position ahead of the true vanishing point (Graf et al., 2007; Jarraya, Amorim, \& Bardy, 2005; Verfaillie \& Daems, 2002; Reed \& Vinson, 1996). Of particular interest here, RM has also been measured for dynamic facial expressions of distinct emotions: fear, joy, disgust, sadness, and anger. Emotional anticipation indexed in terms of RM has been shown to operate either forwards (positive memory bias) or backwards (negative memory bias), depending on the emotion presented and its intensity (Courgeon, Amorim, Giroux, \& Martin, 2010; Yoshikawa \& Sato, 2008), the proposed task (Hubbard, 2005; Kerzel, 2003), the emotional engagement (Palumbo \& Jellema, 2013), and the response timing (Freyd, 1987; see Thornton, 2014). The present study is aimed at detailing the characteristics of this memory bias in the specific context of dynamic facial expressions of pain, through the activation of well-defined expressive features of the face (Prkachin, 1992). Our choice of expressions of pain was motivated by their acknowledged importance as a means to detect other people's pain and to prompt adequate support for the suffering person (Craig, Versloot, Goubert, Vervoort, \& Crombez, 2010; Prkachin, Hughes, Schultz, Joy, \& Hunt, 2002).

Several studies have focused on the contribution of swift automatic processes to the perception of other people's pain, such as emotional contagion and direct matching between other people's pain and self-pain representations (Botvinick et al., 2005; Jackson, Rainville, \& Decety, 2006; Lamm, Batson, \& Decety, 2007). Likewise, we surmise that facial expressions of pain are particularly prone to the automatic anticipation of their future intensity levels. Here, facial expressions are conceived as being characterized by a combination of the type and number of facial muscles contracted, and by the intensity with which they contract (Ekman \& Friesen, 1978). The Facial Action Coding System, also known as FACS (Ekman \& Friesen, 1978), offers a framework for the description of facial expressions in terms of action units of the face (AUs). An observable AU results from the contraction of one or a group of muscle(s), and different facial expressions result from the activation of one or several $\mathrm{AU}(\mathrm{s})$. A facial expression of pain has been shown to recruit three main AUs, namely AU 4, which corresponds to brow lowering, AUs 6 and 7, which correspond to orbit tightening, and AUs 9 and 10, which correspond to levator contraction (nose wrinkling) and upper lip raising (Oliveira, De Sá Teixeira, Oliveira, Breda, \& Da Fonseca, 2007; Prkachin, 1992). The facial expressions of pain employed in our study required the activation of $\mathrm{AU}(\mathrm{s})$ from the upper (AU 4 and $\mathrm{AU}$ 6\&7), the lower (AU 9\&10), or both the upper and the lower parts of the face. Previous studies have indicated that movements in the areas of the eyes, eyebrows, eyelids (e.g., AU 4 and AU 6\&7) and mouth (AU 9\&10), in that order, were rated by observers as being the most important determinants of their judgement of other people's pain (Prkachin, Currie, \& Craig, 1983).

In order to explore the perceptual and memory dynamics of facial pain expressions, we conducted two complementary experiments in this study. In the first experiment, we examined the estimation of other people's levels of pain based on the perception of various dynamic facial expressions; these differed both in terms of the activated AU(s) (i.e., facial expression; FE) and their activation intensity (i.e., expression intensity; IE). In the second experiment, which used a RM paradigm, we investigated the involuntary emotional anticipation (i.e., memory bias) elicited by the same facial expressions of pain used in Experiment 1. These experiments establish the occurrence of an anticipatory memory bias for expressions of pain and its functional dependence on both activated AUs and their activation strength. In addition, we focused on the potential link between perceived pain (addressed in Experiment 1) and the direction and magnitude of the anticipation of a forthcoming emotional state (addressed via the RM in Experiment 2).

\section{Experiment 1}

\section{Method}

In this experiment, we studied the participants' estimation of others' level of pain when perceiving a synthetic character displaying 28 different facial pain expressions that varied according to the activated $\mathrm{AU}(\mathrm{s})$ (i.e., facial expression; FE) and their activation intensity (i.e., expression intensity' EI).

Participants Nineteen participants volunteered to take part in the study (eight females, 11 males; mean age $=27.6$ years, $S D$ $=4.78$ ). Only those with normal or corrected-to-normal vision participated in this experiment. They all first gave their written informed consent. This experiment was conducted in accordance with the Declaration of Helsinki.

Apparatus and stimuli The stimuli used consisted of a set of realistic 3-D synthetic face movements that mobilized specific AUs of pain, and which were created using 3ds Max 2010® software. These movements were implemented on a virtual character imported from the Poser $8 \AA$ software library. In addition to a static neutral expression, seven videos showed a character displaying different dynamic facial expressions and recruiting distinct FACS-defined AUs (Ekman \& Friesen, 1978), as illustrated in Fig. 1. Three facial actions were targeted for modeling, in line with those studied by Oliveira et al. (2007): brow lowering (AU 4), orbit tightening (AUs 6 and 7), comprising "cheek raise" (AU 6) and "lid tightening" (AU 7), and levator contraction (AUs 9 and 10), 


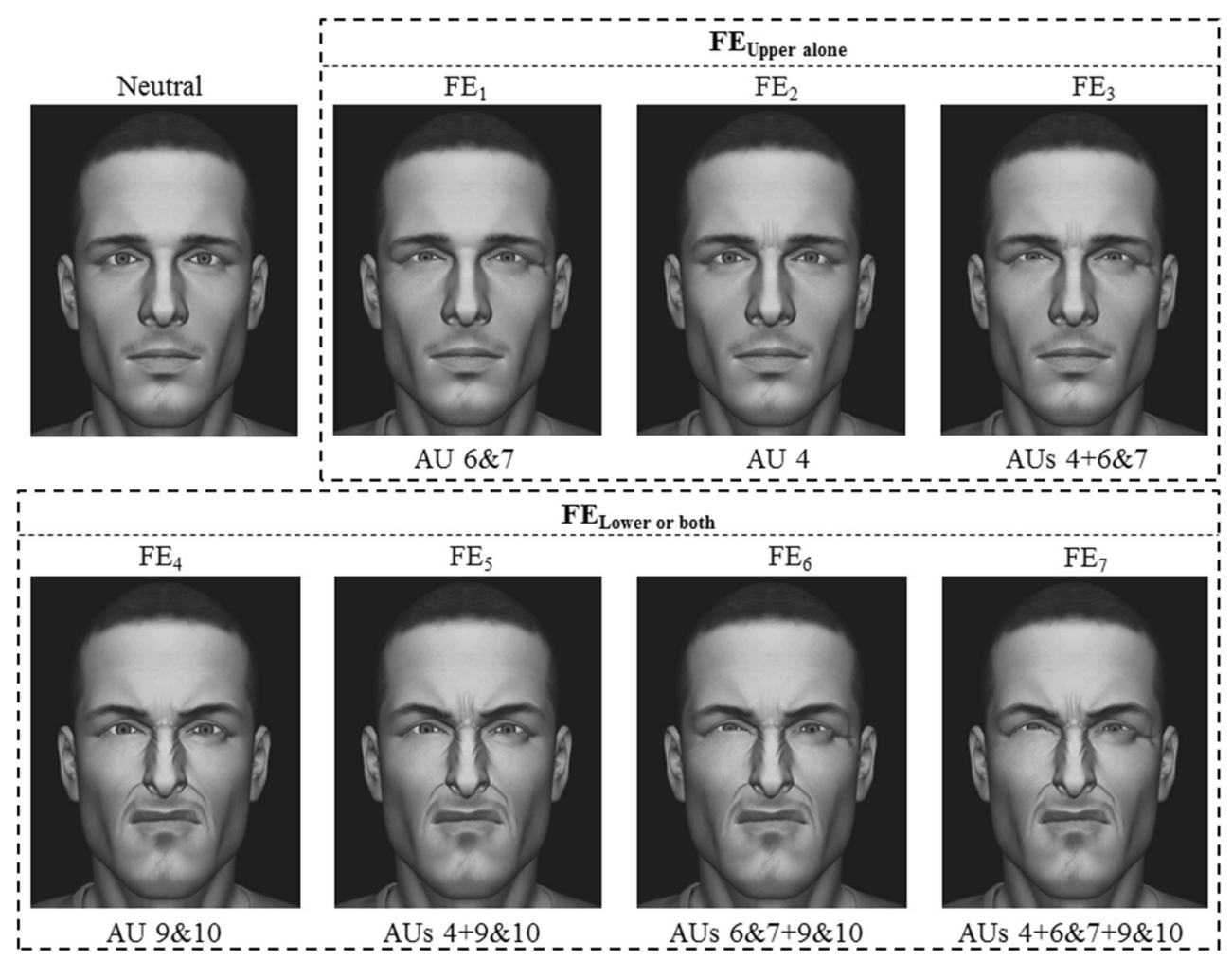

Fig. 1 Illustration of a neutral facial expression, with no action unit (AU) activated, and the seven facial expressions (FE), presented here at maximum intensity $\left(\mathrm{EI}_{100 \%}\right)$. Top row: Neutral facial expression and three expressions called $\mathrm{FE}_{\mathrm{Upper}}$ alone because they only activate the upper face $\mathrm{AU}(\mathrm{s}) . \mathrm{FE}_{1}$ activates $\mathrm{AU} 4$ (brow lowering), causing wrinkles to appear between the eyebrows; $\mathrm{FE}_{2}$ activates $\mathrm{AU} 6 \& 7$ (orbit tightening), causing eyes to close slightly and wrinkles to appear at the outer corners of the eyes; $\mathrm{FE}_{3}$ is a combination of $\mathrm{FE}_{1}$ and $\mathrm{FE}_{2}$. Bottom row: Four expressions called $\mathrm{FE}_{\text {Lower or both }}$ because they either activate lower face $\mathrm{AU}(\mathrm{s})$ alone $\left(\mathrm{FE}_{4}\right)$ or in combination with upper face $\mathrm{AUs}$. $\mathrm{FE}_{4}$ activates AU 9\&10 (levator contraction), causing wrinkles to appear on the nose and above the mouth; $\mathrm{FE}_{5}$ activates AUs 4 and $9 \& 10 ; \mathrm{FE}_{6}$ activates AUs $6 \& 7$ and 9\&10; and $\mathrm{FE}_{7}$ activates these 3 AUs of pain

experiments, and velocity is known to have an effect on automatic anticipation (Actis-Grosso, Bastianelli, \& Stucchi, 2008; Getzmann \& Lewald, 2009; Yoshikawa \& Sato, 2008); thus, we opted to use a constant velocity.

Two experimental factors were manipulated within subjects, with seven and four levels, respectively: Facial expression of pain $\left(\mathrm{FE}_{1}, \mathrm{FE}_{2}, \mathrm{FE}_{3}, \mathrm{FE}_{4}, \mathrm{FE}_{5}, \mathrm{FE}_{6}\right.$, and $\left.\mathrm{FE}_{7}\right)$ and expression intensity $\left(\mathrm{EI}_{25 \%}, \mathrm{EI}_{50 \%}, \mathrm{EI}_{75 \%}\right.$, and $\left.\mathrm{EI}_{100 \%}\right)$. Note that $\mathrm{EI}_{0 \%}$ is not considered to be a level of any of the factors, thus serving as a reference condition (baseline).

Procedure The procedure began with one block of 35 practice trials (not considered for analysis), during which all stimuli were displayed. This practice block allowed participants to familiarize themselves with the task and to calibrate their answers on the response scale. Participants then performed four blocks of 35 trials $(7 \mathrm{FE} \times 5 \mathrm{EI})$. Stimuli $(640 \times 480$ pixels $)$ were displayed at the center of a larger screen $(1,024 \times 768$ pixels), which was positioned at a comfortable distance from the observer (about $57 \mathrm{~cm}$ ). The instructions were: "You will see the face of a person who is experiencing pain. In your opinion, what is the level of pain experienced by this person?" 
Each trial consisted of a stimulus (either a video or a neutral static expression), followed by a horizontal graphic scale anchored on no pain (left) and very strong pain (right), which appeared at the bottom of the screen. Participants had $9 \mathrm{~s}$ to click with a mouse on a chosen location of the scale to express their evaluations, which were registered on a 0-20 format. Stimuli were randomly presented. Participants pressed a keyboard key to start each trial. The experiment lasted about 20 minutes.

\section{Results}

Repeated-measures analyses of variance (ANOVAs) were performed on participants' responses, with the design $7 \mathrm{FE}$ $\times 4 \mathrm{EI}$ and, with the significance threshold set to $p<.05$. The Greenhouse-Geisser correction of degrees of freedom was used whenever sphericity could not be assumed, and corresponding $\varepsilon$ would be indicated. All participants attributed zero value to the pain when the neutral expression was displayed (see Fig. 2, black points at the intersection of the axes). The neutral expression did not actually express pain; thus, it was excluded from the ANOVAs. The results revealed a significant main effect of FE, $F(1.80,32.4)=237.8, \varepsilon=$ $.300, \eta^{2}=.93, \mathrm{MSE}=4.90, p<.0001$, and EI, $F(1.47,26.6)$ $=300.2, \varepsilon=.493, \eta^{2}=.94, \mathrm{MSE}=6.26, p<.0001$, on participants' judgments of conveyed pain. The statistical analysis revealed a significant $\mathrm{FE} \times \mathrm{EI}$ interaction, $F(4.66,83.9)=$ $87.0, \varepsilon=.259, \eta^{2}=.83, \mathrm{MSE}=1.29, p<.0001$.

Visual analysis of the global patterns (see Fig. 2) clearly showed that the seven FE fall into two different categories. The three $\mathrm{FE}_{\text {Upper alone }}$ expressions $\left(\mathrm{FE}_{1}, \mathrm{FE}_{2}\right.$, and $\mathrm{FE}_{3}$; see Fig. 2, solid lines) were associated with low scores of perceived pain, while the four $\mathrm{FE}_{\text {Lower or both }}$ expressions $\left(\mathrm{FE}_{4}\right.$, $\mathrm{FE}_{5}, \mathrm{FE}_{6}$, and $\mathrm{FE}_{7}$; see Fig. 2, dotted lines) were associated with high scores of perceived pain. The $\mathrm{FE}_{\mathrm{Upper}}$ alone $\mathrm{ex}-$ pressions corresponded to subtle feature deformations on the upper part of the face, around the eyes, inducing a smaller increase in rated pain with EI compared with $\mathrm{FE}_{\text {Lower or both. This corresponded to lower face deforma- }}$ tions around the mouth and the nose (either alone or in association with upper face deformations). Moreover, the four lower face expressions, which induced high scores, always included AU 9\&10 (i.e., deformation around the mouth and nose).

Tukey's HSD post hoc tests were performed to decompose the significant FE $\times$ EI interaction. Worth noting, in particular, is the finding that for each EI condition (except $\mathrm{EI}_{25 \%}$ ), mean values of $\mathrm{FE}_{\text {Lower or both }}$ differed significantly from those of

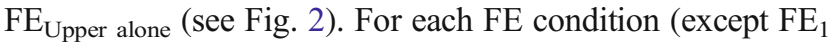
and $\mathrm{FE}_{2}$ ), mean values of each EI were significantly different. For $\mathrm{FE}_{1}$ and $\mathrm{FE}_{2}$, only mean values of $\mathrm{EI}_{75 \%}$ and $\mathrm{EI}_{100 \%}$ differed significantly from those of $\mathrm{EI}_{25 \%}$. Finally, mean perceived pain was significantly different from zero for all FE starting at $\mathrm{EI}_{25 \%}$, including $\mathrm{FE}_{1} \cdot \mathrm{EI}_{25 \%}, t(18)=2.11, p<.05$, associated with the lowest mean perceived pain.

\section{Discussion}

The results of the first experiment indicate that the participants took into account both sorts of visual information (FE and EI) when estimating other people's expressed levels of pain. Participants were thus able to detect the presence of slight deformations ("traces" of pain) in the target faces. In fact, they estimated a level of perceived pain that was significantly different from zero even when only a low intensity of contraction around the eyes was displayed. It is possible that the dynamic character of displayed expressions facilitated the detection of slight variations in conveyed pain (Ambadar, Schooler, \& Cohn, 2005; Bould \& Morris, 2008; Bould, Morris, \& Wink, 2008). In fact, it has been shown that dynamic information proper, rather than the extra static views afforded by a motion sequence, is responsible for the recognition advantage of moving faces (Lander \& Bruce, 2003; Pilz, Vuong, Bülthoff, \& Thornton, 2011; Thornton \& Kourtzi, 2002). Furthermore, for most of the seven FE, participants were capable of significantly discriminating between the four expression intensities. This was true for even the rather subtle expression $\mathrm{FE}_{3}$, which consisted of the activation of the two upper face AUs (AUs 4 and 6\&7). The outcomes of our study also indicated that changes in the lower face led to higher perceived levels of pain than changes in the upper face. This is in agreement with the finding by Oliveira et al. (2007), according to which AU 9\&10 made the greatest contribution to an increase in the ratings of perceived pain. This first experiment supports that individuals present an important capacity to detect subtle facial pain expressions and slight variations (in term of AU and intensity) in pain expressions. It provides new results consistent with Oliveira et al. (2007) showing that upper face AUs (precisely $\mathrm{AU} 6 \& 7$, here $\mathrm{FE}_{1}$ ) are more specifically used to estimate the naturalness of others' pain. This finely tuned perception is necessary because people learn to partially suppress or control their facial display of pain to hide vulnerability (Peeters \& Vlaeyen, 2011) or to avoid embarrassing others (Williams, 2002). Moreover, people can simply try to dupe others by displaying feigned pain expressions. This capacity to detect subtle facial pain expression might be useful to detect feigned or controlled pain expressions.

The advantages offered by dynamic faces include involuntary emotional anticipation (Jellema et al., 2011), which may contribute to the detection of the subtlest pain expressions by automatically anticipating their next step in intensity. Given these results, this should be particularly useful for the lower EIs in the $\mathrm{FE}_{\mathrm{Upper}}$ alone. On the other hand, given the limited range of intensity variation admissible before an unnatural expression occurs, the magnitude of anticipation may be 


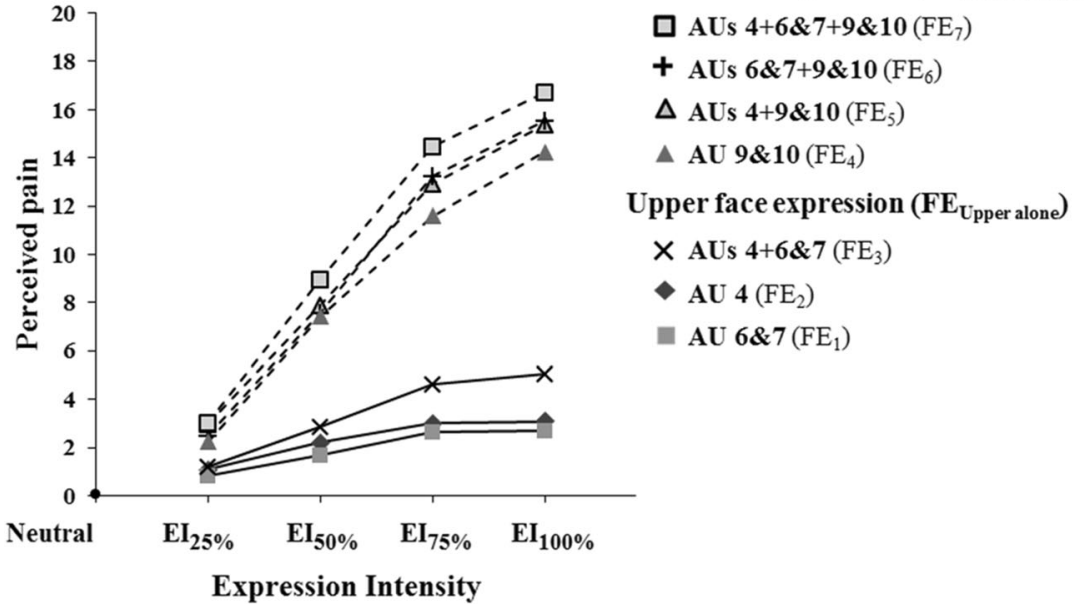

Fig. 2 Perceived pain (mean ratings) as a function of expression intensity (EI, in the abscissa) and facial expression (FE, as the curve parameter) of participants. Perceived pain was always zero when the expression was neutral (see black points at the axes intersection)

expected to decrease as the presented intensities get higher, and even to reverse in direction for the strongest expressions (higher EIs). This would be analogous to the finding of a decrease in representational momentum when the moving target approaches a boundary (Hubbard \& Motes, 2005) or of a negative displacement when the target vanishes just before a rebound collision with an obstacle (Hubbard \& Bharucha, 1988).

The following experiment used a representational momentum (RM) paradigm to highlight the involuntary extrapolation of the immediate future of dynamic pain expressions (same FEs) and check these predictions. By examining how the same sorts of visual information used in the current experiment (i.e., activated AUs and degree of AU activation) modulate the magnitude of RM, it seeks more generally to explore the relationships between obtained ratings of perceived pain and the magnitude and direction of emotional anticipation effects. Finally, by measuring the RM associated with expressions of pain, we can expect to gain access to the properties of the perceiver's inner representation of facial pain specifically rooted in the "perceptual history" of facial expressions (Jellema et al., 2011). This approach, commonly neglected, is based on the idea of dynamic representation as one that includes time as an intrinsic dimension (Freyd, 1987).

\section{Experiment 2}

\section{Method}

In this experiment, we studied the participants' memorization of 18 dynamic facial pain expressions intensities, varying according to the activated AU(s) (i.e., facial expression; FE) and their activation intensity (i.e., expression intensity; EI). With an RM paradigm, we measured the involuntary extrapolation of the immediate future of these dynamic pain expressions.

Participants Thirteen new participants volunteered for the study (four females, nine males; mean age $=27.2$ years, $S D$ $=3.93$ ). Only those with normal or corrected-to-normal vision took part in the experiment. Participants were first required to give their signed informed consent. This experiment was conducted in accordance with the Declaration of Helsinki.

Apparatus and stimuli In this experiment, we used the same dynamic facial expressions (FE) as in our first experiment, except for $\mathrm{FE}_{6}$. The mean perceived pain levels estimated by participants for $\mathrm{FE}_{6}$ and $\mathrm{FE}_{5}$ were similar; thus, we decided to remove $\mathrm{FE}_{6}$ from the design and increase the number of trial replications (to four). The six remaining dynamic expressions were used, with three levels of expression intensity (EI) for each of them $\left(\mathrm{EI}_{50 \%}, \mathrm{EI}_{70 \%}\right.$, and $\left.\mathrm{EI}_{90 \%}\right)$, giving rise to a total of 18 different pain expressions. Moreover, we created static expressions (Test.EI) to be used as memory probe conditions after the presentation of each dynamic expression. For each of the 18 dynamic facial expressions we produced five Test.EI: $-30 \%,-15 \%, 0 \%,+15 \%,+30 \%$, with percentages referring to the intensity of the final expression intensity in the video (EI). Static expressions differed from the preceding dynamic expression only in terms of intensity. For example, in the $\mathrm{FE}_{1}$ condition with $50 \%$ intensity $\left(\mathrm{FE}_{1} \cdot \mathrm{EI}_{50 \%}\right)$ there were five possible static displays of $\mathrm{FE}_{1}$, corresponding to the following five intensities: $20 \%, 35 \%, 50 \%, 65 \%$, and $80 \%$.

Three experimental factors were manipulated within subjects with six, three, and five levels, respectively: Facial expression of pain $\left(\mathrm{FE}_{1}, \mathrm{FE}_{2}, \mathrm{FE}_{3}, \mathrm{FE}_{4}, \mathrm{FE}_{5}\right.$, and $\left.\mathrm{FE}_{7}\right)$, expression intensity $\left(\mathrm{EI}_{50 \%}, \mathrm{EI}_{70 \%}\right.$, and $\mathrm{EI}_{90 \%}$ ), and test expression intensity (Test.EI ${ }_{-30 \%}$, Test.EI ${ }_{-15 \%}$, Test.EI $\mathrm{I}_{0 \%}$, Test.EI $_{+15 \%}$, 
and Test.EI $+30 \%$ ). The three levels of EI were chosen in order to allow for realistic values of Test.EI, with the consequence that two of them did not match the EI values used in Experiment 1. More precisely, $\mathrm{EI}_{100 \%}$ could not be used as it would imply $\mathrm{EI}_{130 \%}$ for Test.EI $\mathrm{I}_{+30 \%}$, which corresponds to an unnatural, nonrealistic facial expression. Similarly, a video with $\mathrm{EI}_{25 \%}$ would entail $\mathrm{EI}_{-5 \%}$ intensity for Test.EI $-30 \%$, which, again, would be a nonrealistic expression. As in the first experiment, video duration depended on the EI condition. We decided to keep velocity, rather than duration, constant, especially as velocity has been shown to have an effect on the magnitude of representational momentum (i.e., RM magnitude increases along with increases in velocity; Actis-Grosso et al., 2008; Getzmann \& Lewald, 2009; Yoshikawa \& Sato, 2008).

Procedure The procedure began with one block of 10 practice trials (not considered for analysis), which were randomly selected from among all the trials undertaken. This training block allowed participants to familiarize themselves with the task. Participants then performed four blocks (i.e., four repetitions) of 90 trials $(6 \mathrm{FE} \times 3 \mathrm{EI} \times 5$ Test.EI), which were randomly presented. Stimuli $(640 \times 480$ pixels $)$ were displayed in the center of a large screen $(1,024 \times 768$ pixels $)$ positioned at a comfortable distance from the observer (about $57 \mathrm{~cm}$ ). The instructions were: "You will see a video presenting the face of a person who is experiencing pain. At the end of the video, the face will be pixelated for a short time and will be followed by a picture of the same person displaying a static expression. Your task will be to judge whether this expression is more or less intense than the expression you saw at the end of the video, just before the pixelated face." To ensure that the instructions were well understood, we showed each participant a schema that was similar to the one given in Fig. 3, except for the name of the condition.

Each trial started with the word go, which was displayed in the center of a black screen for $1 \mathrm{~s}$. A video was then presented (displaying a facial expression that corresponded to a combination of FE and EI, and with a duration of $500 \mathrm{~ms}, 700 \mathrm{~ms}$, and $900 \mathrm{~ms}$, respectively, for $\mathrm{EI}_{50 \%}, \mathrm{EI}_{70 \%}$, and $\mathrm{EI}_{90 \%}$ ), followed by a mask (a pixelated face that lasted for $250 \mathrm{~ms}$ ) and a static expression (a Test.EI, displayed until the participant gave his response). Participants had $5 \mathrm{~s}$ to respond by pressing "esc" for "less intense" and "enter" for "more intense" on the keyboard. Stimuli were randomly presented. Participants pressed a key to start each trial. The experiment lasted for about 1 hour.

\section{Results}

A $6 \mathrm{FE} \times 3 \mathrm{EI} \times 5$ Test.EI repeated-measures analyses of variance (ANOVA), with proportion of "more intense" responses as the dependent variable and the significance

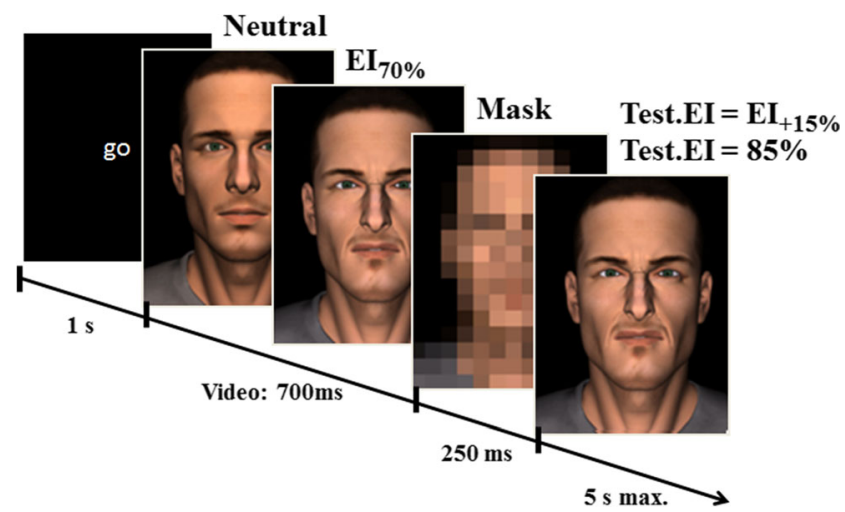

Fig. 3 Schematic illustration of a trial displaying the condition $\mathrm{FE}_{7}$ with $\mathrm{EI}_{70 \%}$ (video from neutral to $70 \%$, with a duration of $700 \mathrm{~ms}$ ) and Test.EI $15 \%$. In this particular trial, Test.EI presents $\mathrm{FE}_{7}$ with an absolute intensity of $85 \%$

threshold set to $p<.05$ revealed significant main effects of FE, $F(1.67,20.1)=19.5, \varepsilon=.33, \eta^{2}=.62$, MSE $=.15$, $p<.0001$; EI, $F(1.33,15.9)=127.2, \varepsilon=.66, \eta^{2}=.91$, MSE $=.05, p<.0001 ;$ and of Test.EI, $F(2.47,29.6)=$ $463.2, \varepsilon=.62, \eta^{2}=.97, \mathrm{MSE}=.06, p<.0001$. Moreover, significant interactions $\mathrm{FE} \times \mathrm{EI} \times$ Test.EI, $F(40,480)=$ $4.1, \eta^{2}=.25, \mathrm{MSE}=.03, p<.0001$; EI $\times$ Test.EI, $F(8$, $96)=14.3, \eta^{2}=.54, \mathrm{MSE}=.03, p<.0001$; and FE $\times$ Test.EI, $F(20,240)=12.8, \eta^{2}=.51$, MSE $=.04, p<$ .0001 (Fig. 4), were also found. The behavior of "more intense" responses as a function of Test.EI was thus shown to differ between $\mathrm{FE}$ and, for each FE, between EI (i.e., the intensity levels at the end of the video).

We used a logistic cumulative distribution (sigmoidal) function to fit the proportion of "more intense" responses, which allowed us to estimate the last-seen facial expression intensity (EI) memorized by participants. For each Test.EI displayed after the mask, participants answered either "less intense" or "more intense" than the final presented EI. The point of subjective equality (PSE) provided an estimate for the Test.EI to which participants would answer "more intense" and "less intense" with an equal 0.50 probability, representing the final memorized intensity expression (Jarraya et al., 2005). PSEs were thus computed as described for each of the 18 facial expressions $(6 \mathrm{FE} \times 3 \mathrm{EI})$ for each participant. Mean memory biases are represented by points in the graph shown in Fig. 5. As can be seen, the mean PSE value for the $\mathrm{FE}_{1} \cdot \mathrm{EI}_{50 \%}$ condition was equal to Test. $\mathrm{EI}_{22 \%}$, indicating that the corresponding memorized expression was $\mathrm{FE}_{1} \cdot \mathrm{EI}_{72 \%}$ (positive bias). A PSE equal to zero corresponds to an accurate mnesic representation of EI (bias equal to 0), a positive PSE to a positive memory bias (forward anticipation) and a negative PSE to a negative memory bias (the represented EI is smaller than the presented EI: backward anticipation). The PSE values, estimated on an individual subject basis, were taken as the dependent variable in a $6 \mathrm{FE} \times 3 \mathrm{EI}$ repeated-measures ANOVA, which disclosed significant main effects of FE, 


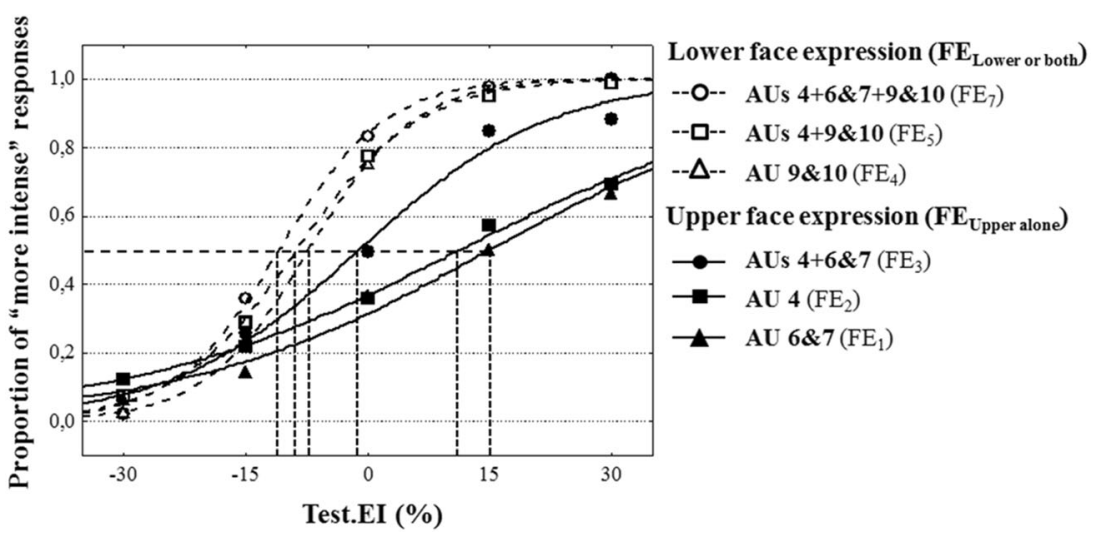

Fig. 4 Proportion of "more intense" responses as a function of Test.EI $(-30 \%,-15 \%, 0 \%,+15 \%,+30 \%)$, with FE as the curve parameter. For each $\mathrm{FE}$, we used a sigmoidal function and estimated the point of subjective equality (PSE) that corresponded to the Test.EI to which participants gave 50\% "more intense" responses (dotted lines). PSE indexes the participants' memory bias. A PSE equal to zero corresponds to an absence of bias, a positive PSE to a positive bias (recalled intensity larger than the one displayed), and a negative PSE to a negative bias (recalled intensity lower than the one displayed)
$F(2.09,25.0)=17.6, \varepsilon=.42, \eta^{2}=.59, \mathrm{MSE}=212.7, p<$ .0001 , and of EI, $F(2,24)=92.4, \eta^{2}=.89, \mathrm{MSE}=85.5, p<$ .0001 . Both sorts of visual information (FE and EI) were thus shown to influence memory bias with a nonsignificant $\mathrm{FE} \times$ EI interaction, $F(3.96,47.5)=1.99, \varepsilon=.39, \eta^{2}=.14$, MSE $=$ 61.1. In addition to the PSE, which measured accuracy, the just noticeable difference (JND) was estimated to assess the precision of the mnesic representation. The JND corresponds to the minimal amount of change required for a difference to become discriminable, thus measuring the participants' sensitivity to changes in EI (Gardner \& Bokenkamp, 1996). The greater the JND, the flatter the curve, reflecting a less precise memory representation of the EI. An additional ANOVA (with the following design: $6 \mathrm{FE} \times 3 \mathrm{EI}$ ) performed on the JNDs revealed a significant main effect of $\mathrm{FE}, F(2.45,29.4)=18.7$, $\varepsilon=.49, \eta^{2}=.61, \mathrm{MSE}=58.8, p<.0001$. Tukey post hoc tests showed that mean JNDs associated with $\mathrm{FE}_{1}$ and $\mathrm{FE}_{2}\left(\mathrm{FE}_{\mathrm{Upper}}\right.$ alone) were significantly superior to those associated with $\mathrm{FE}_{3}$, $\mathrm{FE}_{5}$, and $\mathrm{FE}_{7}$. Mean JND for $\mathrm{FE}_{4}$ differed significantly from the JNDs obtained for $\mathrm{FE}_{1}$ and $\mathrm{FE}_{7}$. These results suggest that $\mathrm{FE}$ - that is, AU(s) activated - can modulate the precision of the participants' memorization of the EI. On the other hand, EI had a nonsignificant main effect, $F(2,24)=.59, \eta^{2}=.04$, MSE $=27.1$, as did the FE $\times$ EI interaction, $F(10,120)=$ $.98, \eta^{2}=.08$, MSE $=41.4$. These results indicate that the expression intensity at the end of the video had no bearing on the precision of the participants' judgments.

\section{Discussion}

The outcomes of Experiment 2 do indeed show a pattern of memory bias (RM) that was modulated both by EI and FE. With regard to EI, the prediction of a decreased RM (which may even turn negative at some point) with increasing

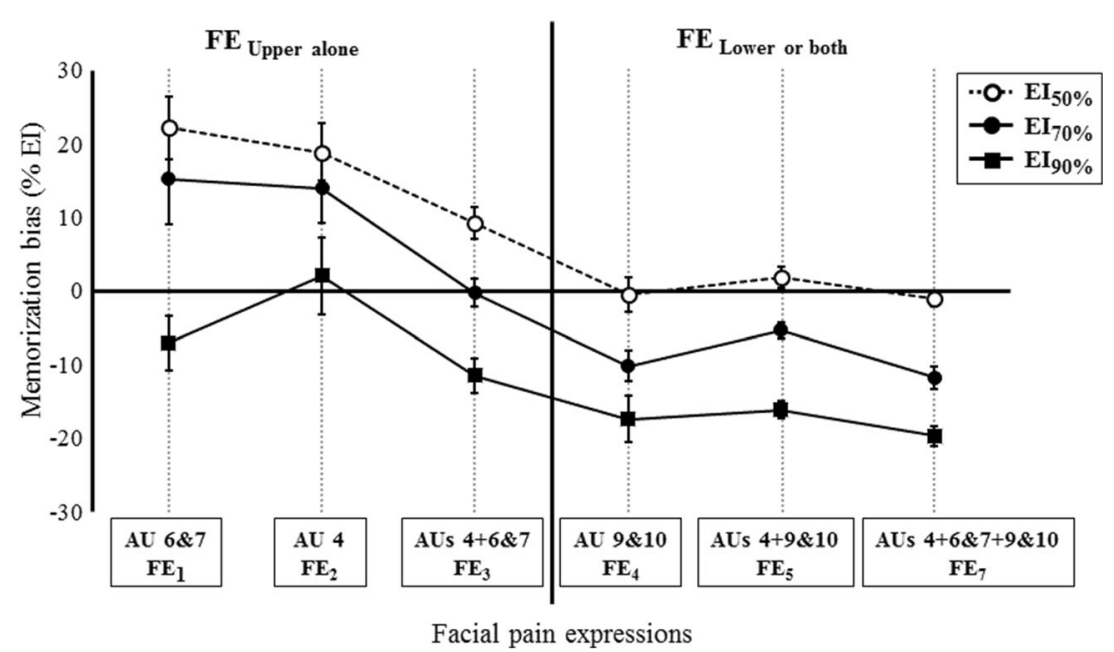

Fig. 5 Pattern of the mean memory biases (on the ordinate) for the 18 facial expressions of pain (on the abscissa). Labels on the horizontal axis specify the FE. EI is the curve parameter. The error bars stand for the standard errors of the mean 
intensities of expression was observed for every FE. Given that increased EI was consistently associated with higher perceptions of facial pain (Experiment 1), this result can justifiably be extended to the relationship between perceived pain intensity and memory bias within each FE, such that larger forward biases are associated with lower perceived intensities and/or larger backward biases with higher perceived intensities. With regard to FE, a clear predominance of positive memory bias in the set of $\mathrm{FE}_{\text {Upper alone }}$ can be seen, contrasting with the predominance of negative bias in the set of $\mathrm{FE}_{\text {Lower or both }}$ (see Fig. 5). This difference matches the one found in Experiment 1 between a group of low-perceived intensity expressions, corresponding to $\mathrm{FE}_{\mathrm{Upper}}$ alone (Fig. 2, solid lines), and a group of high-perceived intensity expressions, corresponding to $\mathrm{FE}_{\text {Lower or both }}$ (Fig. 2, dotted lines), which directly points to a relationship between perceived intensity and the direction of memory bias. This is an important indication that the complete range of variation of perceived pain, and not just a particular range within a given $\mathrm{FE}$, determines the prevalent direction of memory bias. Also, in Fig. 5, we can detect a trend for an overall inverse correspondence between the increased ordering of perceived intensity of the FEs in Experiment 1 (AU 6\&7, AU 4, AUs $4+6 \& 7$, AU 9\&10, AUs $4+9 \& 10$, AUs $4+6 \& 7+9 \& 10$ ) and the ordering of RM values obtained for the same FEs in Experiment 2. Taking into account the RM's decreasing profile across separate FEs for each EI level, it can be seen that, for $\mathrm{EI}_{50 \%}$ and $\mathrm{EI}_{70 \%}$, only one exception to a monotonic decrease with perceived intensity occurs, associated in both cases with FE 5 (AUs $4+9 \& 10$ ). For $\mathrm{EI}_{90 \%}$, a further exception to a monotonic decrease occurs, associated with FE 2 (AU 4). This confirms, in general, the importance of the full range of perceived intensity (across expressions) in determining not just the direction but also, to a large extent, the magnitude of RM. Differently from Experiment 1, the absence of a significant FE $\times$ EI interaction indicates that both sources of information contribute independently (in an adding-type way) to the magnitude of RM.

Taken together, these results are consistent with the idea that perceived intensity plays a major role in determining both the direction and the magnitude of the memory bias (RM). This effect is further mediated by the FEs, which appear to establish a framework for variations in intensity, and the EIs, which determine the variation of perceived intensity within the framework set by each FE. In addition to perceived intensity, however, another factor also appears to be at work in terms of memory bias, as indicated by deviations to a straightforward relationship between perceived intensity and RM and by the cancelation of the FE $\times$ EI interaction when RM, rather than perceived intensity, is the dependent variable. One possible account of these differences would start with the plausible assumption of a nonlinear psychophysical function for perceived intensity.
Local departures from an overall consistent relationship with the memory biases (RM) might thus be expected if the automatic extrapolation mechanism relies not only on perceived intensity but also, as an additional factor, on a tacit understanding of the anatomically-based dynamics of the specific AUs, including the available quantity of deformation between displayed and upper boundary intensities. Taking $\mathrm{FE}_{1}$ as an example, assuming a strongly negatively accelerated psychophysical function for the degree of perceived pain would then render compatible the negligible difference between $\mathrm{EI}_{75 \%}$ and $\mathrm{EI}_{100 \%}$ in Experiment 1 (see Fig. 2) with the large significant difference in RM between levels $\mathrm{EI}_{70 \%}$ and $\mathrm{EI}_{90 \%}$ of $\mathrm{FE}_{1}$ in Experiment 2 (see Fig. 5). A similar reasoning would also apply to $\mathrm{FE}_{2}$ and $\mathrm{FE}_{3}$ for those same intensities (compare Figs. 2 and 5).

A noteworthy feature of the present study is that probes used in RM comparisons among the 18 facial pain expressions $(6 \mathrm{FE} \times 3 \mathrm{EI})$ were selected as equal percentage steps of the morphing range $(15 \%)$. This might be seen as a problem, as our results showed that different facial expressions were not equally discriminable. However, the fact that EI was found to modulate the RM but not the JND, and that no $\mathrm{FE} \times \mathrm{EI}$ interaction emerged for the JND, strongly circumscribes the possible effects of differential discriminability among FEs, thus suggesting that this problem can only (if any) have a limited bearing on the results. Moreover, this memorization of inferior intensity for high facial pain intensities and of superior intensity for low facial intensities could be interpreted as resulting from an averaging process associated with a tendency to reduce high intensity stimuli and increase low intensity ones (Thornton, 2014). Thornton $(1997,2014)$ proposed that some form of memory averaging might be the cause of an observed pattern of backward biases. However, in our study, evidence against this averaging interpretation is provided by comparing $\mathrm{FE}_{3}$ and $\mathrm{FE}_{4}$ in both experiments. In Experiment 1 (see Fig. 2), $\mathrm{FE}_{3} . \mathrm{EI}_{100 \%}$ was associated with a lower mean perceived intensity (5.0) than $\mathrm{FE}_{4} \cdot \mathrm{EI}_{50 \%}$ (7.4). However, in Experiment 2 (see Fig. 5), $\mathrm{FE}_{3} \cdot \mathrm{EI}_{90 \%}$ induced a significant negative bias $(-11.3 \% \mathrm{EI})$, whereas $\mathrm{FE}_{4} . \mathrm{EI}_{50 \%}$ did not result in a significant memory bias $(-0.45 \% \mathrm{EI})$. These last results run counter the averaging interpretation that would have predicted opposite results.

\section{General discussion and conclusion}

Given the pervasiveness of social interactions, individuals have developed specific abilities to perceive and understand other people's facial expressions, namely those of emotion and pain. Some of these abilities may rely on prediction/ anticipation mechanisms, either voluntary or involuntary. At a representational level, Miceli and Castelfranchi (2015) suggested that feeling emotions is often dependent on our 
anticipation of events within a dialectical interaction between "what is" and "what is not (yet)." Thus, the anticipation of future events can induce an emotion (e.g., fear, hope, trust). Furthermore, the confirmation or not of this anticipation can elicit yet other emotions (e.g., surprise, discouragement, regret). The anticipation process can be studied at an explicit representational level (for a review, see Miceli \& Castelfranchi, 2015), but also at a more implicit perceptual level, as in the present study. In our first experiment, we examined the integration of two sorts of visual information in a dynamic facial pain expression. We instructed participants to estimate the level of pain felt by others. In a second experiment, we used a representational momentum (RM) paradigm to investigate the anticipation processes revealed by memory bias when participants are faced with dynamic facial expressions of pain. We used the same facial expressions in both experiments in order to explore the link between the level of perceived pain and the direction and magnitude of emotional anticipation.

The results from Experiment 1 have shown that participants were able to consistently rate perceived pain from even very slight or subtle expressions. According to the literature, individuals have developed the ability to detect facial pain as an adaptive mechanism in order to swiftly perceive and react to other people's pain (Botvinick et al., 2005; Jackson et al., 2006; Lamm et al., 2007). These studies also showed that participants take account of two sources of visual information, which are typically conveyed by an expressive face: the facial action units activated (i.e., FE) and their degree of activation (i.e., EI). The greatest influence on the participants' judgments extended beyond the number of activated AUs to the type of AU itself. In fact, the three facial expressions that were composed only of contractions in the upper face (namely, around the eyes) were all associated with low-perceived pain, whereas those including at least one contraction in the lower face (around the mouth and the nose) induced a high rating of perceived pain. These results are in accordance with those of Oliveira et al. (2007), who showed that contraction in the lower face (AU 9\&10) contributed most to judgments of pain intensity.

In Experiment 2 we addressed the issue of how RM is influenced by the same two kinds of visual pain-related information manipulated in Experiment 1: face area(s) contracted (i.e., FE) and strength of contraction (i.e., EI). RM is known to be influenced by many factors. For moving objects, velocity (Hubbard, 2005), mass, size, and texture of the target (de sá Teixeira, Oliveira, \& Amorim, 2010) have all been shown to affect RM. As for dynamic human faces, the pace of intensity change (Yoshikawa \& Sato, 2008), gaze orientation (Hudson, Liu, \& Jellema, 2009), preceding emotional state (Jellema et al., 2011; Palumbo \& Jellema, 2013) and expressed emotion (Courgeon et al., 2010) have all been found to modulate RM. In the present study, focusing on facial expression components, we explore the effect of two main sorts of expressive information, namely activated action unit(s) (FE) and their intensity of activation (EI) on RM. Our results disclosed an overall relationship between level of perceived pain and the direction and magnitude of the memory bias, such that when perceived pain increased, the memory bias also tended to be reduced (if positive) and ultimately to become negative. Thus, typically, when a facial expression was associated with low ratings of pain, it gave rise to a positive bias with considerable amplitude. Conversely, when a facial expression induced a high rating of perceived pain, it gave rise to a negative bias with a significant magnitude. Courgeon et al. (2010) showed that RM could be negative for high-intensity facial expressions and that RM magnitude could depend on the expressed emotion. We confirmed their results regarding the effect of intensity on the direction of RM while also demonstrating that, even for the same expressed emotion or state (in this case, an expression of pain), both the direction and magnitude of RM were dependent on the facial location of the expressive signal (i.e., the facial area contracted). It is worth noting in this regard that the differential functioning of AUs to the upper and lower face observed in Experiment 1 was also observed in Experiment 2. This constitutes a strong link between the two experiments, because FEs composed of lower facial contractions were predominantly associated with negative bias (remembered intensity lower than the actual one) and FEs composed of upper facial contractions with positive bias (remembered higher intensity than the actual one). We surmise that the cognitive process of automatic anticipation of a facial expression in the future would be most useful when perceiving subtle facial expressions. This interpretation is in accordance with studies showing that individuals preferentially focus their attention on a person's eyes when evaluating the pain they feel (Prkachin et al., 1983), and that the region around the eyes is more heavily weighted when estimating the naturalness of expressions of pain (Oliveira et al., 2007). In this study, we showed that the largest forward anticipation (+22\% of intensity for $\mathrm{FE}_{1} . \mathrm{EI}_{50 \%}$, see Fig. 5) was elicited by the subtlest facial pain expression, which was associated with the lowest level of perceived pain in Experiment 1. This memory bias would thus enable the detection of rapid changes in facial expressions, particularly those that are quite subtle. Given that emotional communication in daily life is based principally on dynamic facial cues, which are most often very subtle (Yoshikawa \& Sato, 2008), this cognitive process would seem to be highly adaptive.

It is important to recall that stimuli displayed in this study were synthetic facial expressions of pain. Furthermore, a second way in which these stimuli are not natural concerns the linear, constant velocity dynamics adopted in the videos. Thornton (2014) showed that very schematic faces did not induce emotional anticipation, in contrast to synthetic virtual facial expressions (Courgeon et al., 2010). One interpretation 
could be that the more information there is in the face, the more facial expressions can prompt emotional anticipation by observers (Thornton, 2014). In the present study, even though a nonrealistic constant velocity of increasing intensity was used, our results suggest that facial expressions were informative enough for the purpose, as participants did exhibit anticipation effects. Furthermore, various studies have shown that human facial expressions mostly leads to backward shifts in memory (Marian \& Shimamura, 2013; Thornton, 2014, 1997), and similar results have also been found with synthetic facial expression (Courgeon et al., 2010). We also observe this pattern in the present study. In fact, among the 18 facial pain expressions displayed, only four have induced a forward shift (positive memory bias; see Fig. 5). As previously mentioned, these four facial expressions are those associated with the lowest perceived level of pain in Experiment 1 (see Fig. 2). Our pattern of results suggests that the more an expression is associated with a high level of perceived pain, the more backward will be its associated memory shift. We surmise that the important number of memory backward shifts reported in the concerned literature could be due to the choice of stimuli. Indeed, we can presume that the displayed endpoints of the videos used corresponded most of the time to high intensities of expression, being thus associated with high levels of perceived pain. Therefore, replicating our experiment with videos of real facial pain expressions involving an equivalent range of perceived intensities while, on the other hand, displaying more ecological expressive dynamics, would surely constitute an interesting prospect for assessing whether, and to which extent, the use of linear synthetic dynamic expressions constrains the results.

In the present study, we focused on the perceptual level of the anticipation process. Proponents of simulation theory and of the embodiment approach to the perception of facial expressions (Niedenthal, Mermillod, Maringer, \& Hess, 2010; Wicker et al., 2003) have suggested that an involuntary motor simulation of the observed facial expression causes the observer to "experience" the observed action, which in turn contributes information about the agent's emotional state. In accordance with previous studies, we assume that dynamic facial expressions of pain begin by reenacting an "immediate perceptual history" in the perceiver before leading to an "emotional anticipation" of the agent's upcoming state (Jellema et al., 2011; Palumbo \& Jellema, 2013). Several studies have shown that other people's pain can prompt emotional contagion and a direct matching of self-pain representations (Botvinick et al., 2005; Jackson et al., 2006; Lamm et al., 2007). Thus, studying an individual's anticipation processes may offer access to the perceivers' inner representations of dynamic pain expressions. Our results suggest that participants have a tacit understanding that the dynamic progress of a facial expression is commonly composed of an onset (increasing), an apex (maximum), and an offset (decreasing) phase. This would be consistent with the finding that facial expressions of pain perceived to convey low levels of pain are anticipated to increase (onset phase), and those perceived to convey high levels of pain anticipated to decrease (offset phase). The negative shift of memorization in case of high intensity of pain could thereby be explained by a boundary effect associated to "a clear maximum extent of facial expression" (Hubbard, 2005, p. 831). A tacit representation of the dynamics of facial pain expressions would also be consistent with the finding that several expressions actually present an associated RM of zero. We surmise that, rather than signaling a lack of anticipation, this absence of memory bias reflects anticipation in the direction of a constant intensity (apex phase). In fact, five out of the 18 presented expressions induced a RM value near zero (see Fig. 5), despite involving different AU combinations at distinct intensities (both physical and perceived). The suggested anticipation of an apex phase for these different cases can be squared with the notion that there is actually not one but several types of pain-for example, physical, emotional, episodic, chronic or constant, and tonic (Prkachin, 1997). Moreover, individuals may express their own pain in different ways: for instance, some individuals typically suppress and control their facial display of pain either to hide vulnerability from others (Karmann, Lautenbacher, Bauer, \& Kunz, 2014; Kunz, Chen, Lautenbacher, Vachon-Presseau, \& Rainville, 2011; Peeters \& Vlayen, 2011) or to avoid embarrassing others (Williams, 2002). Thus, individual differences in the tacit representation of expressions of pain may also manifest themselves in a different relationship between perceived pain and RM-indexed anticipation, which should become a topic of future research.

In summary, our findings provide evidence that the involuntary anticipation of a painful state (indexed by RM) when observing a facial expression of pain can vary both in direction and magnitude, depending on the level of perceived pain, which in turn varies as a function of two kinds of facial visual information: the type and number of $\mathrm{AU}(\mathrm{s})$ activated and the intensity of activation. Future investigations could examine the impact of other types of visual information on RM, such as the velocity at which intensity increases. Magnitude of anticipation in the classical RM effect is known to increase with velocity (Actis-Grosso et al., 2008; Getzmann \& Lewald, 2009; Yoshikawa \& Sato, 2008). Would this also be the case for all kinds of pain expressions, though? It may be the case that some facial expressions of pain mostly reflect acute pain and are thus more sensitive than others to the velocity at which intensity increases.

One may also consider RM behavior for decreases in the intensity of facial expressions (offset phase), rather than increases (onset phase). A dynamic expression that moves from neutral to a smile is judged more positively than when moving from a smile to neutral (Marian \& Shimamura, 2013). An expression that vanishes during the offset phase 
may thus tell a different "immediate perceptual history" (Jellema et al., 2011) and thereby have a distinct effect on the RM. More generally, it may also be considered whether the emotion category and the context in which the expression occurs have an impact on the direction and amplitude of the anticipation. Inasmuch as the context is allowed to partake in the "immediate perceptual history" of the expression (Jellema et al., 2011), nothing opposes in principle that it may influence the ensuing anticipation. For example, the cognitive process of anticipation may differ depending on whether an individual perceives a facial expression of pain in a clinical or a nonclinical setting, and on personal experience of this setting (e.g., being a clinician, an emergency doctor, a therapist). From this point of view, the commonly reported finding that clinicians underestimate patients' pain (Kappesser, Williams, \& Prkachin, 2006; Marquié et al., 2003; Prigent, Amorim, Leconte, \& Pradon, 2014; Prkachin, Solomon, \& Ross, 2007) might correspond to a significantly adaptive anticipation that relates to the particular context and constraints of their job.

Acknowledgements We would like to express our gratitude to the participants.

\section{References}

Actis-Grosso, R., Bastianelli, A., \& Stucchi, N. (2008). Direction of perceptual displacement of a moving target's starting and vanishing points: The key role of velocity. Japanese Psychological Research, 50, 253-263.

Ambadar, Z., Schooler, J. W., \& Cohn, J. F. (2005). Deciphering the enigmatic face the importance of facial dynamics in interpreting subtle facial expressions. Psychological Science, $16,403-410$.

Botvinick, M., Jha, A. P., Bylsma, L. M., Fabian, S. A, Solomon, P. E., \& Prkachin, K. M. (2005). Viewing facial expressions of pain engages cortical areas involved in the direct experience of pain. NeuroImage, $25,312-319$.

Bould, E., \& Morris, N. (2008). Role of motion signals in recognizing subtle facial expressions of emotion. British Journal of Psychology, 99, 167-189.

Bould, E., Morris, N., \& Wink, B. (2008). Recognising subtle emotional expressions: The role of facial movements. Cognition and Emotion, 22, 1569-1587.

Courgeon, M., Amorim, M.-A., Giroux, C., \& Martin, J.-C. (2010). Do users anticipate emotion dynamics in facial expressions of a virtual character? Proceedings of the 23rd Annual Conference on Computer Animation and Social Agents (CASA2010), SaintMalo, France.

Craig, K. D., Versloot, J., Goubert, L., Vervoort, T., \& Crombez, G. (2010). Perceiving pain in others: Automatic and controlled mechanisms. The Journal of Pain, 11, 101-108.

de sá Teixeira, N., Oliveira, A. M., \& Amorim, M.-A. (2010). Combined effects of mass and velocity on forward displacement and phenomenological ratings: A functional measurement approach to the momentum metaphor. Psicologica, 31, 659-676.

Ekman, P., \& Friesen, W. V. (1978). Manual for the Facial Action Coding System. Palo Alto, CA: Consulting Psychologists Press.
Freyd, J. J. (1987). Dynamic mental representations. Psychological Review, 94, 427-438.

Freyd, J. J., \& Finke, R. A. (1984). Representational momentum. Journal of Experimental Psychology: Learning, Memory, \& Cognition, 10, $126-132$.

Gardner, R. M., \& Bokenkamp, E. D. (1996). The role of sensory and nonsensory factors in body size estimations of eating disorder subjects. Journal of Clinical Psychology, 52, 3-15.

Getzmann, S., \& Lewald, J. (2009). Constancy of target velocity as a critical factor in the emergence of auditory and visual representational momentum. Experimental Brain Research, 193, 437443.

Graf, M., Reitzner, B., Corves, C., Casile, A., Giese, M., \& Prinz, W. (2007). Predicting point-light actions in real-time. NeuroImage, $36(2), 22-32$.

Hubbard, T. L. (2005). Representational momentum and related displacements in spatial memory: A review of the findings. Psychonomic Bulletin \& Review, 12, 822-851.

Hubbard, T. L. (2014). Forms of momentum across space: Representational, operational, and attentional. Psychonomic Bulletin \& Review, 21, 1371-1403.

Hubbard, T. L., \& Bharucha, J. J. (1988). Judged displacement in apparent vertical and horizontal motion. Perception \& Psychophysics, 44, 211-221.

Hubbard, T. L., \& Motes, M. A. (2005). An effect of context on whether memory for initial position exhibits a Fröhlich effect or an onset repulsion effect. Quarterly Journal of Experimental Psychology, 58, 961-979.

Hudson, M., Liu, C. H., \& Jellema, T. (2009). Anticipating intentional actions: The effect of eye gaze direction on the judgment of head rotation. Cognition, 112, 423-434.

Jackson, P. L., Rainville, P., \& Decety, J. (2006). To what extent do we share the pain of others? Insight from the neural bases of pain empathy. Pain, 125, 5-9.

Jarraya, M., Amorim, M.-A., \& Bardy, B. G. (2005). Optical flow and viewpoint change modulate the perception and memorization of complex motion. Perception \& Psychophysics, 67, 951-961.

Jellema, T., Pecchinenda, A., Palumbo, L., \& Tan, E. G. (2011). Biases in the perception and affective valence of neutral facial expressions induced by the immediate perceptual history. Visual Cognition, 19, 616-634.

Kappesser, J., Williams, A. C., \& Prkachin, K. M. (2006). Testing two accounts of pain underestimation. Pain, 124, 109-116.

Karmann, A. J., Lautenbacher, S., Bauer, F., \& Kunz, M. (2014). The influence of communicative relations on facial responses to pain: Does it matter who is watching? Pain Research \& Management, 19, $15-22$.

Kerzel, D. (2003). Attention maintains mental extrapolation of target position: Irrelevant distractors eliminate forward displacement after implied motion. Cognition, 88, 109-131.

Kunz, M., Chen, J. L., Lautenbacher, S., Vachon-Presseau, E., \& Rainville, P. (2011). Cerebral regulation of facial expressions of pain. Journal of Neurosciences, 31, 8730-8738.

Lamm, C., Batson, C. D., \& Decety, J. (2007). The neural basis of empathy: Effects of perspective taking and cognitive appraisal. Journal of Cognitive Neuroscience, 19, 42-58.

Lander, K., \& Bruce, V. (2003). The role of motion in learning new faces. Visual Cognition, 10, 897-912.

Marian, D. E., \& Shimamura, A. O. (2013). Contextual influences on dynamic facial expressions. American Journal of Psychology, 126, $53-65$.

Marquié, L., Raufaste, E., Lauque, D., Mariné, C., Ecoiffier, M., \& Sorum, P. (2003). Pain rating by patients and physicians: Evidence of systematic pain miscalibration. Pain, 102, 289-296.

Miceli, M., \& Castelfranchi, C. (2015). Expectancy and emotion. New York, NY: Oxford University Press. 
Niedenthal, P. M., Mermillod, M., Maringer, M., \& Hess, U. (2010). The Simulation of Smiles (SIMS) model: Embodied simulation and the meaning of facial expression. Behavioral and Brain Sciences, 33, 417-433.

Oliveira, A. M., De Sá Teixeira, N. A., Oliveira, M. P., Breda, S. J., \& Da Fonseca, I. (2007). Algebraic integration models of facial features of expression: A case made for pain. Teorie \& Modelli. Rivista di Storia e Metodologia della Psicologia, 12(1/2), 155-166.

Palumbo, L., \& Jellema, T. (2013). Beyond face value: Does involuntary emotional anticipation shape the perception of dynamic facial expressions? PLOS ONE, 8(2), e56003.

Peeters, P., \& Vlaeyen, J. (2011). Feeling more pain, yet showing less: The influence of social threat on pain. Journal of Pain, 12, 12551261.

Pilz, K. S., Vuong, Q. C., Bulthoff, H. H., \& Thornton, I. M. (2011). Walk this way: Approaching bodies can influence the processing of faces. Cognition, 118, 17-31.

Prigent, E., Amorim, M. A., Leconte, P., \& Pradon, D. (2014). Perceptual weighting of pain behaviours of others, not information integration, varies with expertise. European Journal of Pain, 18, 110-119.

Prkachin, K. M. (1992). The consistency of facial expressions of pain: A comparison across modalities. Pain, 51, 297-306.

Prkachin, K. M. (1997). The consistency of facial expression of pain: A comparison across modalities. In P. Ekmand \& E. Rosenber (Eds.), What the face reveals (pp. 181-197). Oxford, UK: Oxford University Press.

Prkachin, K. M., Currie, N. A., \& Craig, K. D. (1983). Judging nonverbal expressions of pain. Canadian Journal of Behavioural Science, 15, 409-421.
Prkachin, K. M., Hughes, E., Schultz, I., Joy, P., \& Hunt, D. (2002). Realtime assessment of pain behavior during clinical assessment of low back pain patients. Pain, 95, 23-30.

Prkachin, K. M., Solomon, P. E., \& Ross, J. (2007). Underestimation of pain by health-care providers: Towards a model of the process of inferring pain in others. Canadian Journal of Nursing Research, 39, 88-106.

Reed, C. L., \& Vinson, N. G. (1996). Conceptual effects on representational momentum. Journal of Experimental Psychology: Human Perception and Performance, 22, 839-850.

Thornton, I. M. (1997). The perception of dynamic human faces (Doctoral dissertation, University of Oregon, Eugene). Retrieved from http://www.ianthornton.com/publications/

Thornton, I. M. (2014). Representational momentum and the human face: An empirical note. Xjenza, 2(2), 9, 101-110. https://doi.org/10. 7423/XJENZA.2014.2.09

Thornton, I. M., \& Kourtzi, Z. (2002). A matching advantage for dynamic human faces. Perception, 31, 113-132.

Verfaillie, K., \& Daems, A. (2002). Representing and anticipating human actions in vision. Visual Cognition, 9, 217-232.

Wicker, B., Keysers, C., Plailly, J., Royet, J.-P., Gallese, V., \& Rizzolatti, G. (2003). Both of us disgusted in my insula: The common neural basis of seeing and feeling disgust. Neuron, 40, 655-664.

Williams, A. C. C. (2002). Facial expression of pain, empathy, evolution, and social learning. Behavioral and Brain Sciences, 25, 475-480.

Yoshikawa, S., \& Sato, W. (2008). Dynamic facial expressions of emotion induce representational momentum. Cognitive, Affective, \& Behavioral Neuroscience, 8, 25-31. 